Case Report

\title{
Unruptured saccular aneurysm presenting migraine
}

\author{
M. Zhao, C.S. Liu, X.Y. Xu, Y.P. Xiao and C. Fang \\ Department of Neurology, Shanghai Pudong New Area Zhoupu Hospital, \\ Shanghai, China \\ Corresponding author: M. Zhao \\ E-mail: meizhaocn@126.com
}

Genet. Mol. Res. 13 (2): 4046-4049 (2014)

Received July 24, 2013

Accepted November 29, 2013

Published January 24, 2014

DOI http://dx.doi.org/10.4238/2014.January.24.19

\begin{abstract}
Headache can be attributed to cranial or cervical vascular disorders including ischemic stroke or transient ischemic attack, nontraumatic intracranial hemorrhage, unruptured vascular malformation, arteritis, carotid-vertebral artery pain, and cerebral venous thrombosis. Here, we present a case report of unruptured saccular aneurysm with migraine. The patient was a previously healthy 32 -year-old man with repeated episodes of headache for 6 years. Findings for computed tomography and magnetic resonance imaging of the head were normal. Head magnetic resonance angiography revealed a small, nodule-like protuberance seen in the anterior communicating artery. Based on his clinical features and ancillary examinations, the patient was diagnosed with migraine without aura. The patient subsequently underwent digital subtraction angiography (DSA), which revealed a 2-mm cystic protuberance in the superoposterior anterior communicating artery. The patient underwent stent-assisted coil embolization of the aneurysm. Subsequent DSA results indicated no recurrence of aneurysm and no recurrence of headache was reported after surgical treatment.
\end{abstract}

Key words: Unruptured saccular aneurysm; Migraine attack; Headache 


\section{INTRODUCTION}

Headache may be attributed to cranial or cervical vascular disorders that include (listed by code number) ischemic stroke or transient ischemic attack, non-traumatic intracranial hemorrhage, unruptured vascular malformation, arteritis, carotid-vertebral artery pain, and cerebral venous thrombosis. It is important not to miss or misdiagnose disorders that can cause headaches and may lead to death. Here, we describe a patient with an unruptured saccular aneurysm of an atypical size that showed no signs of cranial nerve or pyramidal tract injury. To the best of our knowledge, similar cases have not been reported in the literature.

\section{CASE REPORT}

On November 17, 2011, a 32-year-old male patient was admitted to our hospital because of repeated episodes of headache for 6 years. The headache occurred in an episodic pattern, with a pulsating and throbbing character. The patient experienced pain in the periorbital and temporal regions, and the headache attacks were accompanied by flu-like symptoms, including lacrimation, nasal congestion, rhinorrhea, and facial sweating. Sometimes, the pain was accompanied by pallor and blurred vision. Each attack lasted from several hours to 1-2 days and was likely triggered or aggravated by fatigue and stress. During an attack, the patient preferred a quiet environment, and rest could relieve the symptoms. During some attacks, the patient experienced severe and unbearable pain that was accompanied by nausea, vomiting, blurred vision, and fatigue, which could be relieved with intramuscular injection of bucinnarizine. The frequency of the migraines was uncertain, but they typically occurred once or twice per month. The patient visited his local hospital regularly, and took ibuprofen and codeine for pain relief. On November 9, 2011, the patient visited the outpatient clinic of the Department of Neurology of East Hospital. An initial examination revealed normal findings for head computed tomography $(\mathrm{CT})$ and magnetic resonance imaging, a small, nodule-like protuberance in the anterior communicating artery (Figure 1A). Digital subtraction angiography (DSA) was used to confirm the diagnosis, and the patient was admitted to our hospital for further examination. On physical examination, the patient was conscious and had fluent speech. He had no cranial nerve abnormalities, and his orientation, memory, and mathematical ability were normal. Muscle strength and tone in the limbs were normal and without atrophy or resting tremor. Superficial and deep sensations in the limbs were symmetrical. His reflexes were normal and without pathological signs. He did not have neck stiffness, and Brudzinski's and Kernig's signs were absent. During admission, he was diagnosed with migraine without aura. Laboratory tests yielded normal blood, urine, and stool findings. Moreover, plasma glucose levels, kidney function, levels of electrolytes, and serum lipid levels were normal. Human immunodeficiency virus antibody and syphilis TRUST tests were both negative. However, the patient was positive for hepatitis B core antibody and hepatitis B surface antibody and negative for hepatitis C antibody. No abnormalities were seen on electroencephalography or electrocardiography. An increased number of lung markings were noted bilaterally on X-ray radiography of the chest.

The patient underwent DSA on November 18, 2011. Under local anesthesia, the right femoral artery was punctured, and sheath placement was performed. A guidewire-guided $5 \mathrm{~F}$ vertebral artery catheter was used to perform arterial angiography in the internal carotid, subclavian, and vertebral arteries bilaterally. A 2-mm cystic protuberance was identified in 
the superoposterior anterior communicating artery; no significant stenosis or expansion was identified in other intracranial vessels (Figure 1B). The brain was stained homogeneously in the parenchymal phase, and the reflux was smooth in the venous phase. On November 23, 2011, the patient underwent stent-assisted coil embolization of the aneurysm under general anesthesia using an Enterprise 4.5 x 14-mm stent (Cordis Neurovascular Inc., Miami Lakes, FL, USA). Bilateral femoral artery puncture was performed, and the sheath was placed. A guidewire was used to place the $6 \mathrm{~F}$ and $5 \mathrm{~F}$ guiding catheters in the $\mathrm{C} 2$ segment of the internal carotid arteries. Angiography was performed, and firmly adhered to the arterial wall. The majority of the aneurysm was embolized (Figure 1C). Head CT after surgery showed no significant abnormalities in the brain parenchyma. After surgery, oral aspirin enteric-coated tablets $(100 \mathrm{mg} /$ day $)$ and clopidogrel (75 mg/day) were given.

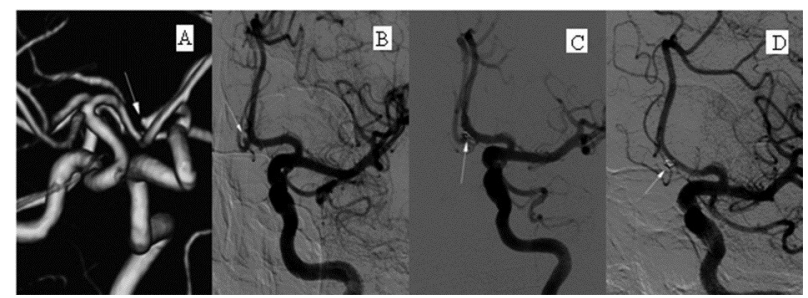

Figure 1. Relative saccular aneurysm images (white arrow pointing at the lesion). A. Head magnetic resonance angiography; B. Digital subtraction angiography (DSA) before stent-assisted coil embolization; C. DSA after stentassisted coil embolization; D. DSA in follow-up.

On January 9, 2012, the patient was readmitted for examination. On January 11, 2012, a right femoral artery puncture was performed using Seldinger's technique, and a 5F arterial sheath was placed for conventional cerebral angiography. The DSA results indicated no recurrence of aneurysm following embolization of the anterior communicating artery aneurysm (Figure 1D), and the stent was patent and firmly adhered to the arterial wall. Oral aspirin enteric-coated tablets $(100 \mathrm{mg} /$ day $)$ and clopidogrel $(75 \mathrm{mg} /$ day $)$ were continued. No recurrence of headache was reported after surgical treatment.

\section{DISCUSSION}

The patient was a previously healthy 32-year-old man with no history of any other disease. The disease course was chronic, and the clinical features were recurrent headache accompanied by lacrimation, nasal congestion, rhinorrhea, pallor, and blurred vision. No neurological signs were noted on physical examination. According to his clinical features and ancillary examinations, the patient's provisional diagnosis was confirmed as migraine without aura. The patient met the diagnostic criteria of the International Classification of Headache Disorders, 2nd edition (ICHD-2), established by Headache Classification Subcommittee of the International Headache Classification Subcommittee of the International Headache Society (2004). The patient had been taking ibuprofen and codeine to relieve his pain and distress from frequent migraines and chronic headache. To date, the patient has experienced no further episodes of headache following the interventional surgery. Therefore, the association between headache and surgery is noteworthy. The patient was discharged with a diagnosis of symptomatic migraine. 
Aneurysms larger than $10 \mathrm{~mm}$ can predispose the patient to thrombosis, or may cause local meningeal irritation leading to headache. Cranial nerve injury, pyramidal tract signs, and visual acuity or field disorders may be other symptoms of large aneurysms. Headache accompanied by oculomotor nerve palsy is the most common feature in such patients. Mydriasis can present larger aneurysms or those increasing in size, which is an early sign of oculomotor nerve compression at the junction between the internal carotid artery and posterior communicating artery, at the junction between the basilar artery and superior cerebellar artery, and at the tip of the basilar artery. The size of the unruptured saccular aneurysm in our patient was atypical, and the patient showed no signs of cranial nerve or pyramidal tract injury. The patient was examined with follow-up for 8 months after stent-assisted coil embolization of the aneurysm, and no recurrent episode of headache was noted. To our knowledge, no similar case has been reported in the literature (Bigal et al., 2004; Holroyd et al., 2007; Brandes, 2008; Wang et al., 2008); thus, further study is required to explore the underlying mechanisms involved. Early diagnosis and aggressive intervention may not only reduce the risk of the aneurysmal rupture, but also effectively control headache. We plan to conduct long-term follow-up for this patient. In conclusion, our report suggests that the relationship between unruptured saccular aneurysm and migraine is worthy of further investigation.

\section{ACKNOWLEDGMENTS}

We are grateful for the helps from W. Xie and R. Ojha.

\section{REFERENCES}

Bigal ME, Lipton RB and Stewart WF (2004). The epidemiology and impact of migraine. Curr. Neurol. Neurosci. Rep. 4: 98-104.

Brandes JL (2008). The migraine cycle: patient burden of migraine during and between migraine attacks. Headache 48: 430-441.

Headache Classification Subcommittee of the International Headache Society (2004). The international classification of headache disorders: 2nd edition. Cephalalgia 1: 1-160.

Holroyd KA, Drew JB, Cottrell CK, Romanek KM, et al. (2007). Impaired functioning and quality of life in severe migraine: the role of catastrophizing and associated symptoms. Cephalalgia 27: 1156-1165.

Wang XP, Ding HL, Geng CM and Jiang YM (2008). Migraine as a sex-conditioned inherited disorder: evidences from China and the world. Neurosci. Bull. 24: 110-116. 